\title{
The long-term activity of dwarf novae and soft X-ray transients
}

\author{
Vojtěch Šimon* \\ Astronomical Institute, The Czech Academy of Sciences, 25165 Ondřejov, Czech Republic \\ Czech Technical University in Prague, Faculty of Electrical Engineering, Prague, Czech \\ Republic \\ E-mail: simondasu.cas.cz
}

\begin{abstract}
We show the activity of dwarf novae (DNe) and soft X-ray transients (SXTs) which were observed to undergo series of relatively frequent outbursts. The length of the recurrence time of these events, $T_{\mathrm{C}}$, is usually less than a year (at most about 4 years). We show that the energy output (e.g. fluence) sometimes largely differs even for the neighboring outbursts both of DNe and SXTs. This energy output is related to the fraction of the disk mass, accreted during an outburst, but this difference of the energy output does not influence the length of $T_{\mathrm{C}}$ in a given object. The length of $T_{\mathrm{C}}$ in a given DN or SXT is variable, but the occurrence of these outbursts is not dramatically irregular in most cases. The long-term trends can be clearly resolved in the $\mathrm{O}-\mathrm{C}$ diagrams of $T_{\mathrm{C}}$ but they show only small epoch-to-epoch fluctuations. In DNe, the disk zone where the outburst starts can change even in a given binary for the individual outbursts. The decaying branch of the outburst is governed by propagation of cooling front and the observations show that this part of the light curve reproduces well for the ensemble of outbursts in a given DN. As for SXTs, the final phase of the decay in the 1.5-12 keV band reproduces well for the individual outbursts while the preceding phase can vary for the individual events. We also show an analogy of the ZCam-type behavior in an SXT (transitions between the segments of a hot thermally stable (in standstills) and unstable disk (outbursts)).
\end{abstract}

The Golden Age of Cataclysmic Variables and Related Objects - IV,

11-16 September 2017

Palermo, Italy

* Speaker. 


\section{Introduction}

Dwarf novae (DNe) (a type of cataclysmic variables (CVs)) are binary systems containing a mass-accreting white dwarf (WD) (see e.g [37] for a review). DNe display strong outbursts that are interpreted in terms of a thermal-viscous instability (TI) of the accretion disk (e.g. [25, 9]). The optical brightness of DNe strongly rises during the outburst (typically by $2-5 \mathrm{mag}$ ), which is caused by a transition of the disk from the cool state to the hot state. This leads to accretion of matter from the disk onto the WD.

Soft X-ray transients (SXTs) are similar to DNe but they contain the neutron star (NS) or the black hole (BH) instead of the WD (see e.g. [20] for a review). They undergo outbursts similar to those in DNe. However, the conditions in the disks of SXTs can be affected by the presence of a very hot inner disk region; it can act as an irradiating source during outburst and is thought to modify the disk structure (e.g. [6]).

The long-term activity of a vast majority of DNe is observed only in the optical band by the available instruments. On the other hand, the long-term activity of SXTs is observed mostly in X-rays.

\section{The outbursting activity of dwarf novae (DNe)}

\subsection{GK Per}

GK Per (A 0327+43) is an intermediate polar [38]. It suggests that the WD is magnetized and this magnetic field influences the accretion flow. Nowadays, it undergoes 2-3 mag optical DN outbursts with the recurrence time $T_{\mathrm{C}}$ of hundreds of days (e.g. [23, 12, 28]). Since GK Per has a very long orbital period $P_{\text {orb }}$ of 1.99 days [4], hence a large accretion disk, it has a long DN outbursts. This enables us to determine their profiles in detail.

The light curve of the time segment in which the DN outbursts occurred is displayed in Fig. 1a. The data are from AFOEV, AAVSO [11, 14], and [31]. The parameters of the outbursts of GK Per are often largely unstable, different even for the consecutive events. Often the outbursts with the fainter peak magnitude are also shorter; this suggests less energy emitted by the disk in a given outburst. A segment of the outbursts with the mutually similar parameters consists only of 8 consecutive events (Figs. 1bc).

The DN outbursts are discrete events. The method of the $\mathrm{O}-\mathrm{C}$ residuals thus appears suitable for their analysis. The method of using the $\mathrm{O}-\mathrm{C}$ residuals of some reference period is widely used in the study of the orbital period changes of eclipsing binaries but only rarely applied to investigation of outbursts in DNe. [35] used this method for a study of the superoutbursts in the SU UMa subtype and long outbursts in two U Gem-type CVs. This method enables us not only to determine $T_{\mathrm{C}}$, but also to analyze its variations. It works with the residuals from some reference period (i.e. with the deviations from a constant period). This method is not sensitive to the exact length of the reference period because a slightly different reference period gives rise only to an additional linear trend of the $\mathrm{O}-\mathrm{C}$ curve, which can be corrected later. The resulting $\mathrm{O}-\mathrm{C}$ curve also enables us to assess the position of each outburst with respect to the $\mathrm{O}-\mathrm{C}$ profile of the remaining outbursts. A relation between the $\mathrm{O}-\mathrm{C}$ curve and $T_{\mathrm{C}}$ is as follows: a linear profile of the $\mathrm{O}-\mathrm{C}$ curve (regardless of the slope) suggests a constant $T_{\mathrm{C}}$; a parabolic profile of $\mathrm{O}-\mathrm{C}$ means a linear change of $T_{\mathrm{C}}$. 

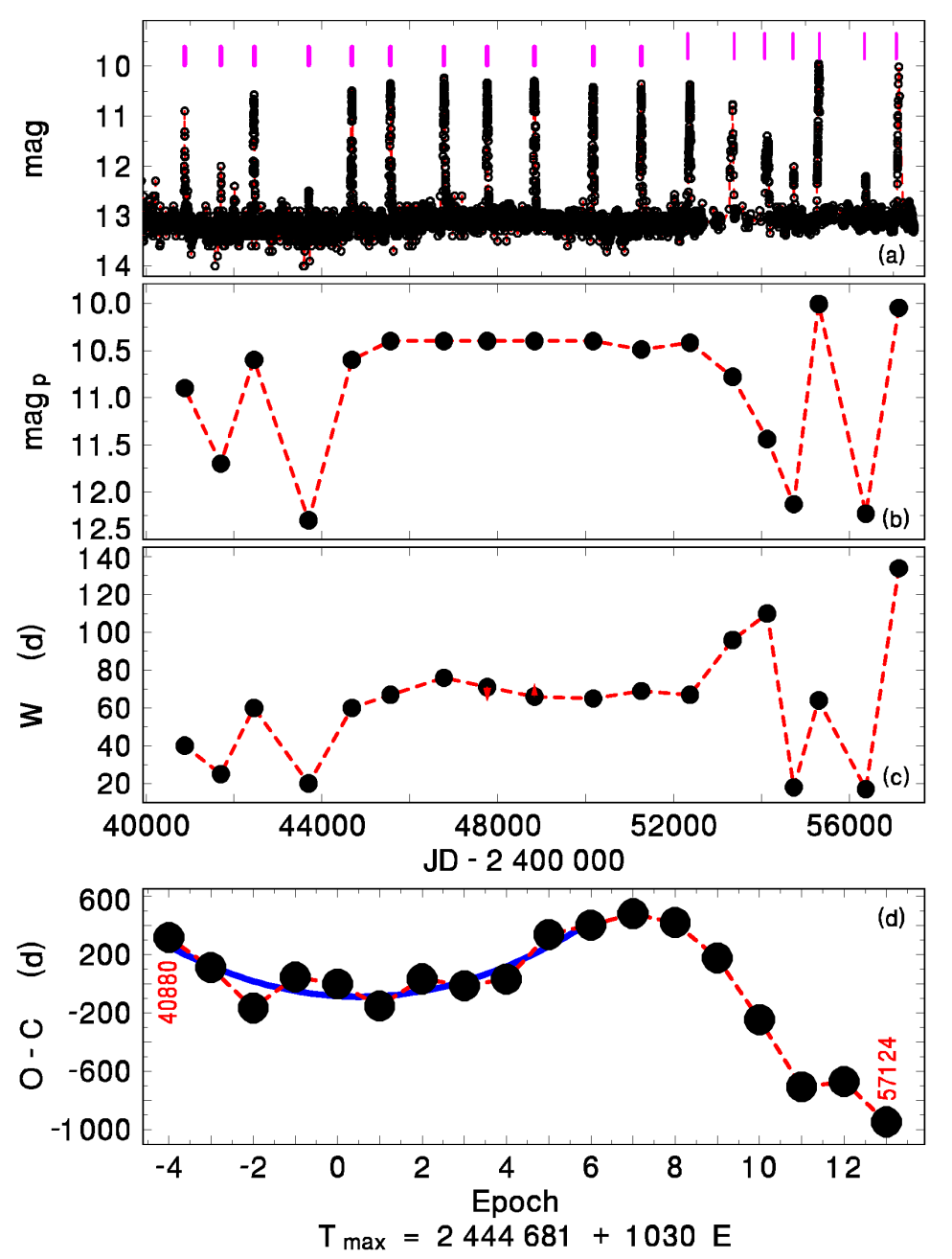

Figure 1: (a) Light curve of GK Per (mostly one-day means). The time segment in which the DN outbursts occurred is displayed. The individual outbursts are marked by the vertical lines. (b, c) Evolution of the parameters of the DN outbursts. The peak magnitude is abbreviated as $\operatorname{mag}_{\mathrm{p}}$. The width of the outburst is marked as $W$ (in days). (d) $\mathrm{O}-\mathrm{C}$ diagram of $T_{\mathrm{C}}$. The numbers at some points represent the times of the outburst peaks in JD-2 400000 for convenience. Also a fit by a second degree polynomial is shown. The points are connected by a line to guide the eye.

Figure 1 shows that neither the peak magnitude of the outburst nor the width of the outburst are related to the current length of $T_{\mathrm{C}}$ in GK Per. Variations of $T_{\mathrm{C}}$ are large, but not dramatically irregular (Fig. 1d). The duration of the quiescent interval (prior to or after the outburst) is thus largely independent of the amount of the radiated energy of a given outburst. This suggests that the properties of the disk in the cool state play a big role.

The light curves of the DN outbursts in GK Per are displayed in Fig. 2. Notice that the decaying branch of the DN outburst remains remarkably similar for all the events. The decaying branches of the individual outbursts were merged into a common file and smoothed by the code HEC13, written by Prof. P. Harmanec [10]. It is based on the method of [36], who improved the original method of 
Whittaker [39].

The decaying branch of the outburst is a measure of propagation of cooling front. This part of the DN outburst reproduces well for the individual events. This suggests that all the decays are controlled by the same process with very similar parameters. On the other hand, the large changes of the light curve can occur on the rising branch. The light curves of some outbursts, especially of those with the steep rising branch, also display a viscous plateau (a roughly flat top).

The variable slopes of the rising branches can be explained in the framework of the TI model [25] in the following way. In the case that the onset of a TI occurs in the outer part of the disk, the outburst is outside-in. Its light curve has a steep rise. A TI of the inside-out outburst, starting in the inner parts of the disk and propagating outwards, produces instead a more symmetric light curve. The location of the onset of a TI can depend also on the drift of matter during quiescence. In the framework of a TI, we interpret Fig. 2 in terms of heating front which starts at a different radial distance from the center of the disk for the individual events. The further from the center of the disk the onset of a TI occurs, the more the outburst resembles the outside-in type.

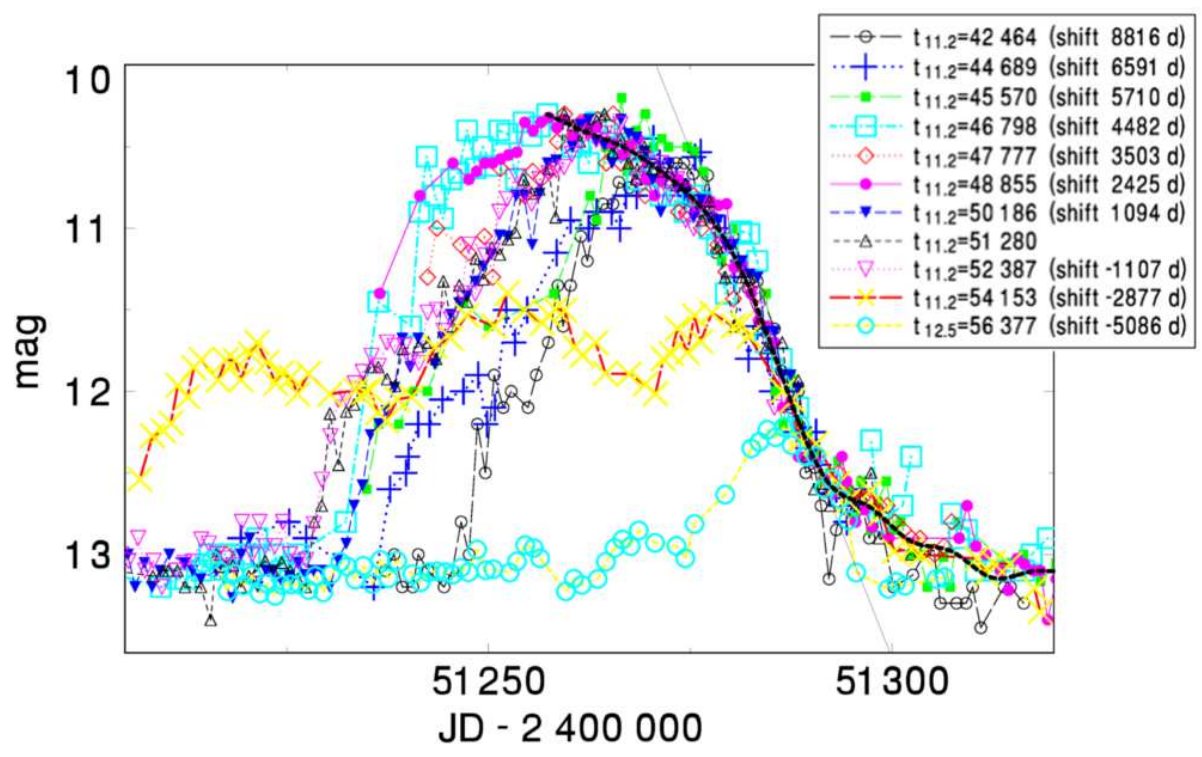

Figure 2: The DN outbursts in GK Per. The one-day means of a given outburst were connected by a line. The individual outbursts were shifted along the time axis to match the decaying branch of the template. The time of crossing $11.2 \mathrm{mag}$ and the shifts with respect to the template are listed. The thick curve represents the smoothed curve. The tilted straight line denotes the decaying branch which can be considered as linear in the magnitude scale.

\subsection{CH UMa}

CH UMa (a U Gem type CV [17]) exhibits several types of the DN outbursts [30, 26]. Also it belongs to CVs with a rather long $P_{\text {orb }}$ (8.28 hours) [32].

Our previous analysis [26] showed that the statistical distribution of the peak brightness is bimodal and the outbursts can be divided into two classes, the division magnitude being 12 mag. The 
updated light curve is displayed in Fig. 3a. The bright outbursts fall into two subtypes according to their decaying branches (fast and slow decays).

Since CH UMa is circumpolar for the observers in the Northern Hemisphere, it is reasonable to suppose that the dense observing series contains all outbursts brighter than $12 \mathrm{mag}$. We used the data from AFOEV and AAVSO $[11,14]$. Figure $3 \mathrm{~b}$ shows the O-C diagram of $T_{\mathrm{C}}$ of the outbursts brighter than 12 mag.

Large $\mathrm{O}-\mathrm{C}$ changes of the bright outbursts were found, larger than the reference period. One therefore has to be cautious in constructing the $\mathrm{O}-\mathrm{C}$ curve. The parts of the $\mathrm{O}-\mathrm{C}$ curve which contained the large variations therefore were examined using several reference period lengths to obtain the smallest slope of the curve in the vicinity of the change. Although the $\mathrm{O}-\mathrm{C}$ curve mostly shows gradual variations, also several jumps are visible. The outbursts are even absent in some time segments, especially in the surroundings of JD 2456500 . These gaps also lead to the jumps in the $\mathrm{O}-\mathrm{C}$ curve.
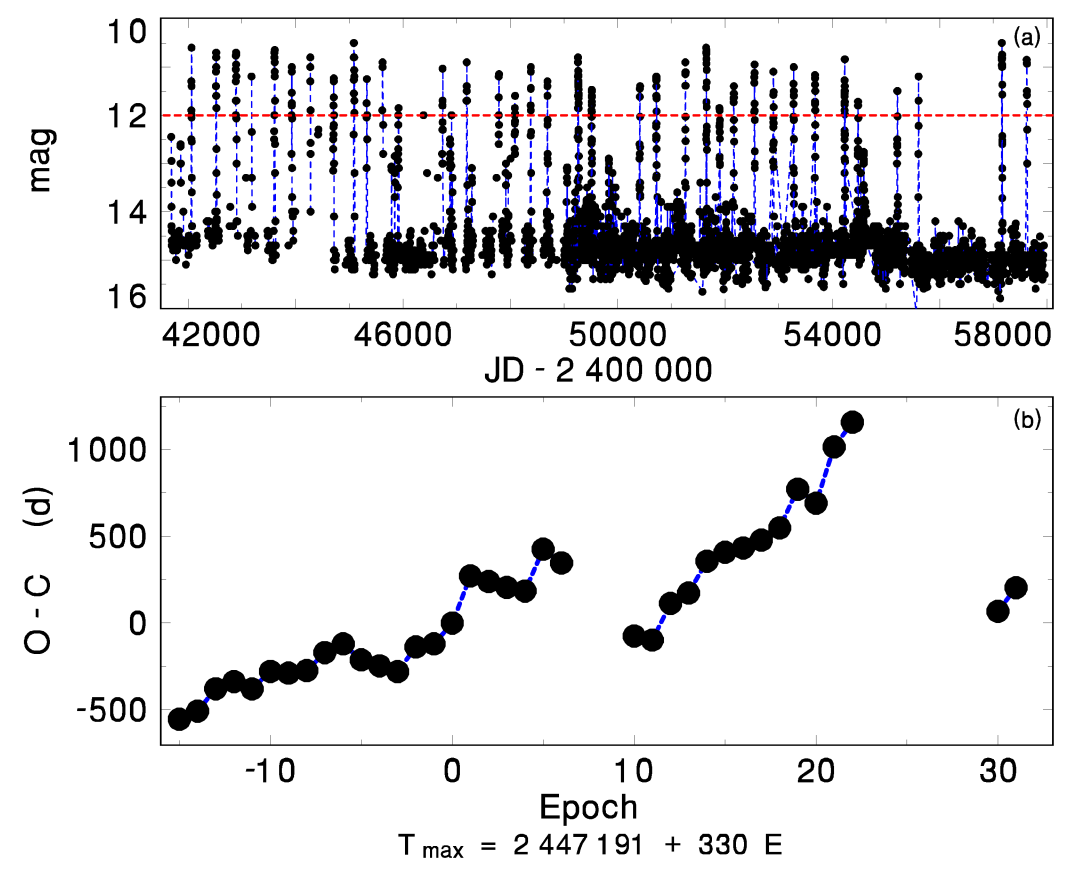

Figure 3: (a) Light curve of CH UMa (mostly one-day means). The horizontal line at 12 mag separates the group of the bright outbursts from the faint outbursts. (b) $\mathrm{O}-\mathrm{C}$ diagram of $T_{\mathrm{C}}$. The points are connected by a line to guide the eye.

\subsection{Ser}

X Ser (HV 3137) and its classical nova outburst in 1903 were discovered by Leavitt on Harvard plates [18, 7]. Its $P_{\text {orb }}$ is $1.478 \mathrm{~d}$ [33]. The light curve shows that the episodic brightenings with the peak-to-peak amplitude of about 2 mag occurred in the post-nova [8].

This analysis uses the $V$-band CCD data [14] and the Catalina Real-time Transient Survey observations [5]. Figure 4 shows a comparison of the individual episodic brightenings of the postnova. These events were aligned according to their decaying branches. The decaying branches of 
the individual outbursts were merged into a common file and smoothed by the above-mentioned code HEC13.

The decay rate reproduces itself well for the set of these outbursts. We interpret these events as the DN outbursts (see Sect. 2.4 for more). The rises of these events are quite slow, which implies inside-out type of outburst. In the interpretation, the largely different peak magnitudes of the individual events suggest that heating front may not reach the outer disk rim in some outbursts. More details are given in [29].

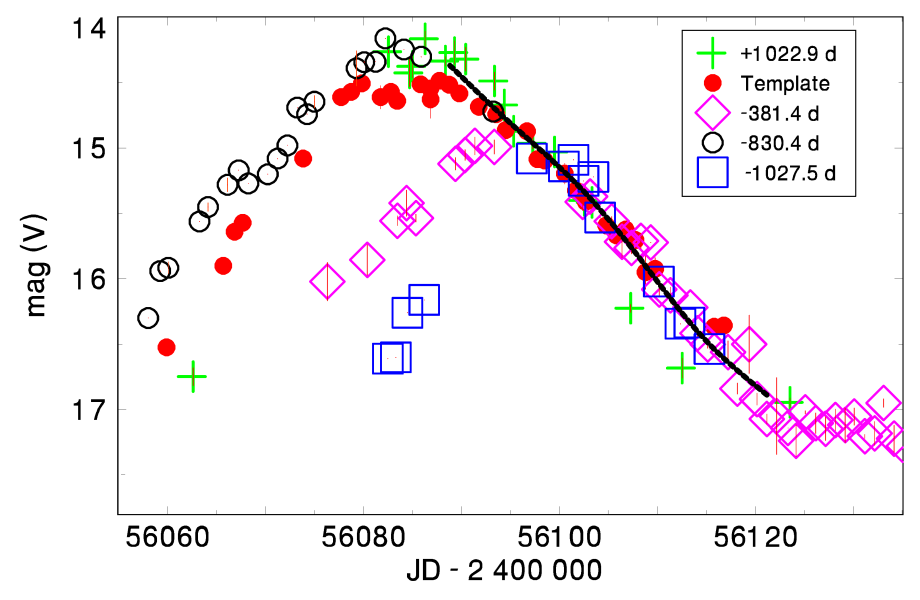

Figure 4: Comparison of the DN outbursts in X Ser. The events were aligned according to their decaying branches to match the decaying branch of the template. The time of crossing $15.5 \mathrm{mag}(V)$ and the time shifts with respect to the template are listed. The thick dashed curve represents the mean smoothed curve of the decaying branch.

\subsection{The decay rate of the DN outbursts}

The decay rate is an important parameter of the light curves of the DN outbursts. The quantity $\tau_{\mathrm{D}}$ is the decay rate expressed in days for a decrease of brightness by 1 mag [1].

Positions of various DNe are shown in Fig. 5 where $\tau_{\mathrm{D}}$ is plotted versus $P_{\text {orb }}$. This plot enables to assess how much a given outburst obeys the Bailey relation [1]. The data come from [37], [24], [27] (DO Dra); the data for UY Pup were determined from the AAVSO database [14]. Please note that the values of $\tau_{\mathrm{D}}$ of DODra and GK Per are smaller than of most DNe with the comparable $P_{\text {orb }}$. These two CVs are known to be the intermediate polars according to [21, 38].

\section{Outbursting activity of selected soft X-ray transients (SXTs)}

The long-term activity of the soft X-ray transients (SXTs) is mostly observable only by the X-ray monitors. The characteristic features (e.g. outbursts) can be investigated even in a singleband X-ray light curve (monitors sometimes work with a single band observing (typically in soft and medium X-rays, with the energy $E$ of a few $\mathrm{keV}$ )). Even some model predictions are already available, e.g. as regards the modification of the light curve of the outburst and $T_{\mathrm{C}}$ by a possible irradiation of the disk [6]. 


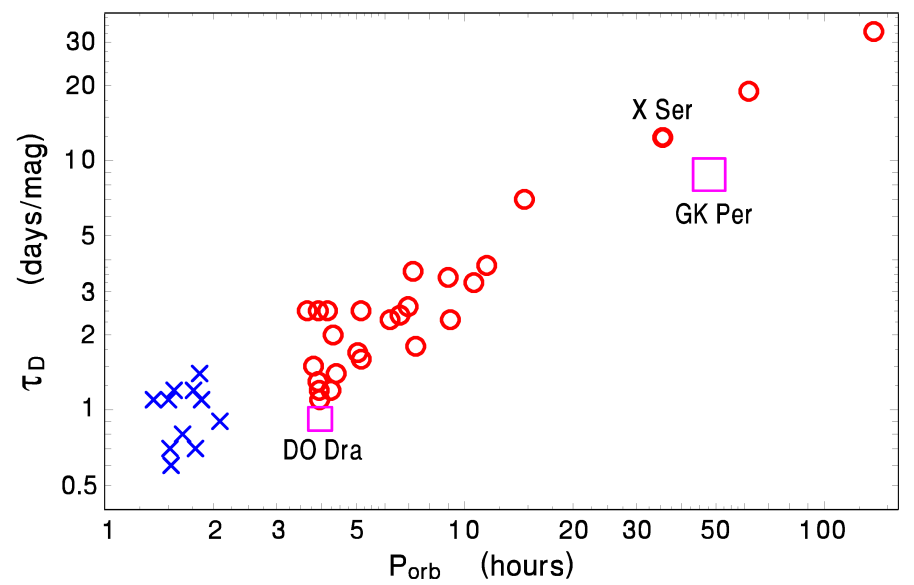

Figure 5: Decay rate of the DN outbursts, $\tau_{\mathrm{D}}$, versus $P_{\text {orb }}$. DNe below and above the period gap (e.g. [37]) are included. The crosses represent the SU UMa type. The squares mark the intermediate polars.

\subsection{Aql X-1/V1333 Aql}

Aql X-1 is a low-mass X-ray binary (LMXB) which contains the NS [16] and the K6-M0 mass-donating companion [3]. Its $P_{\text {orb }}$ is 18.71 hours [2].

We used the observations obtained by RXTE/ASM [19]. Figure 6a shows that the outbursts display large variations of their peak flux. Even the consecutive outbursts can have very different fluences (Fig. 6b) (fluence is the flux integrated over the duration of the whole outburst). Variations of $T_{\mathrm{C}}$ are large, but the occurrences of the outbursts are not random (Fig. 6c).

The previous analyses of $T_{\mathrm{C}}$ of the outbursts of Aql X-1 were based on the automated period search. [22] carried out Fourier analysis of the events which occurred in 1969-1975. They argued that there is an underlying cycle of 123 days which does not lead necessarily to every outburst. They also admitted a phase smearing of the outbursts. [15] analyzed the timings of outbursts which occurred in 1969-1992. They used the method of calculating the standard deviation of observed phases from a trial period. They concluded that the original $T_{\mathrm{C}}$ changed from 125 days to 309 days. Our analysis shows that the length of $T_{\mathrm{C}}$ displays episodes both of a decrease and increase of the length. These episodes and time segments are independent of the strength (fluence) of the previous or the following outbursts.

Figure 7 shows that the profile of the decaying branch (the measure of propagation of cooling front) reproduces well for the individual outbursts. Its slope is largely independent of the peak luminosity of the outburst. The decaying branch shows no clear sign of an exponential decay. In the model of [6], an exponential decay is a sign that the disk is irradiated during the outburst. The profiles of the decaying branches in Fig. 7 therefore speak against irradiation of the disk in these outbursts of Aq1 X-1.

\subsection{HETE J1900.1-2455}

HETE J1900.1-2455 is a low-mass X-ray binary (LMXB) with the NS accretor [34, 13]. Its $P_{\text {orb }}$ is very short, only $83.3 \mathrm{~min}$, the companion can be a lobe-filling brown dwarf [13]. 

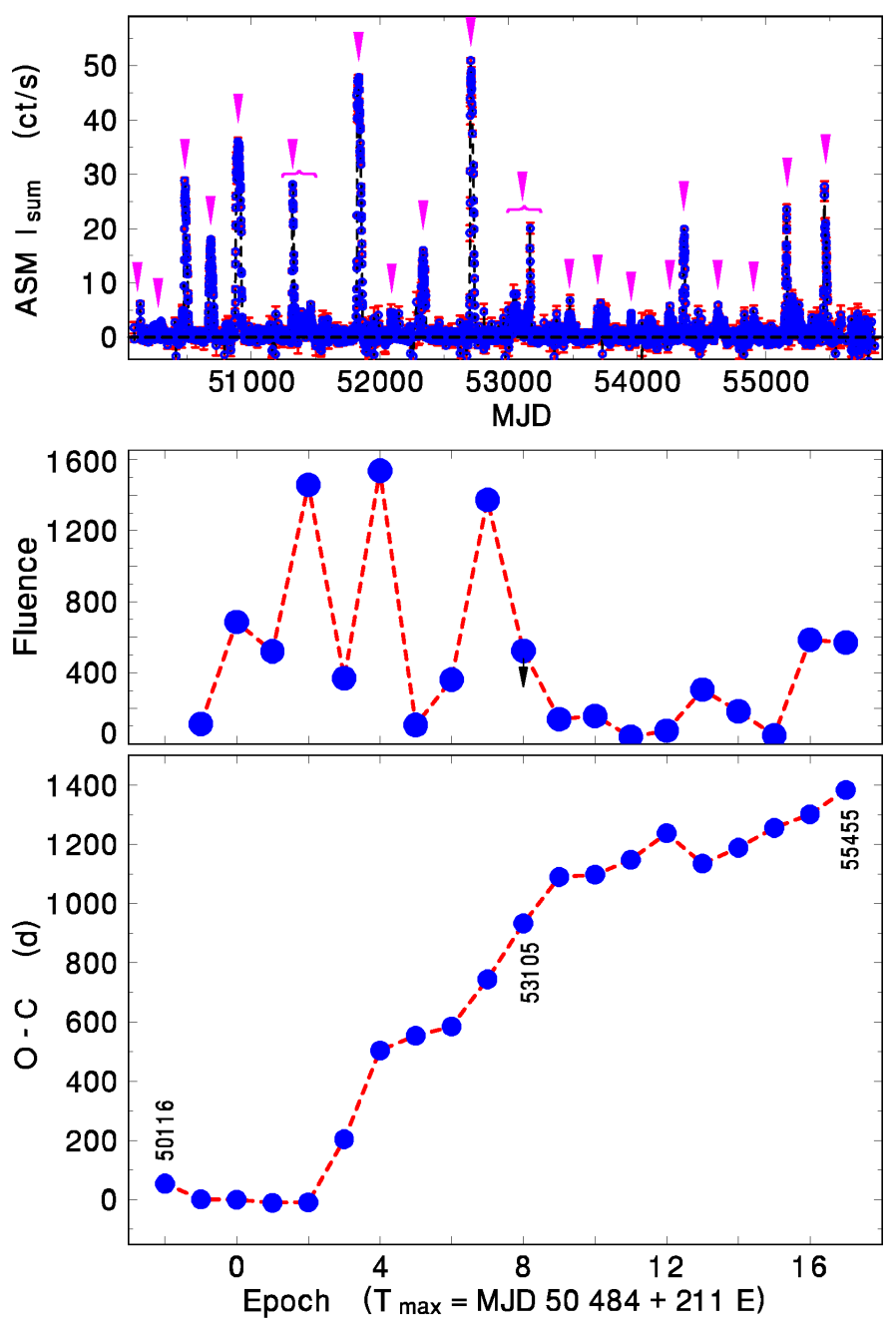

Figure 6: (a) Light curve of Aql X-1 in the 1.5-12 keV band (the one-day means of the RXTE/ASM observations). The individual outbursts are marked. (b) Fluences of the individual outbursts. (c) O-C diagram of $T_{\mathrm{C}}$. The numbers at some points represent the times of the outburst peaks in MJD for convenience. The points are connected by a line to guide the eye.

The MAXI light curve in the $2-4 \mathrm{keV}$ band in Fig. $8 \mathrm{a}$ shows a series of brightenings from a faint baseline level. The duration of each such brightening is several days, their cycle-length is about 50 days. These features are similar to the closely spaced outbursts of SXTs. This outbursting activity of HETE J1900.1-2455 is from time to time interrupted by the segments in which the flux remains roughly between that of the peak of the outburst and the baseline of the outbursts.

Such segments bear similarity to the so-called standstills observed in the optical band in the ZCam DNe. The standstills are the high-state episodes with the ionized disk on the hot branch of the S-curve of a TI [37]. The peaks of their DN outbursts are brighter than these standstills. For comparison, an example of the optical activity of the Z Cam-subtype of DNe is displayed in Fig. 8b.

In conclusion, the activity of HETE J1900.1-2455 can be a link between the activity of the NS and the WD accreting with the mass transfer rate which oscillates between an operating TI of the disk and the disk residing in the ionized state. 


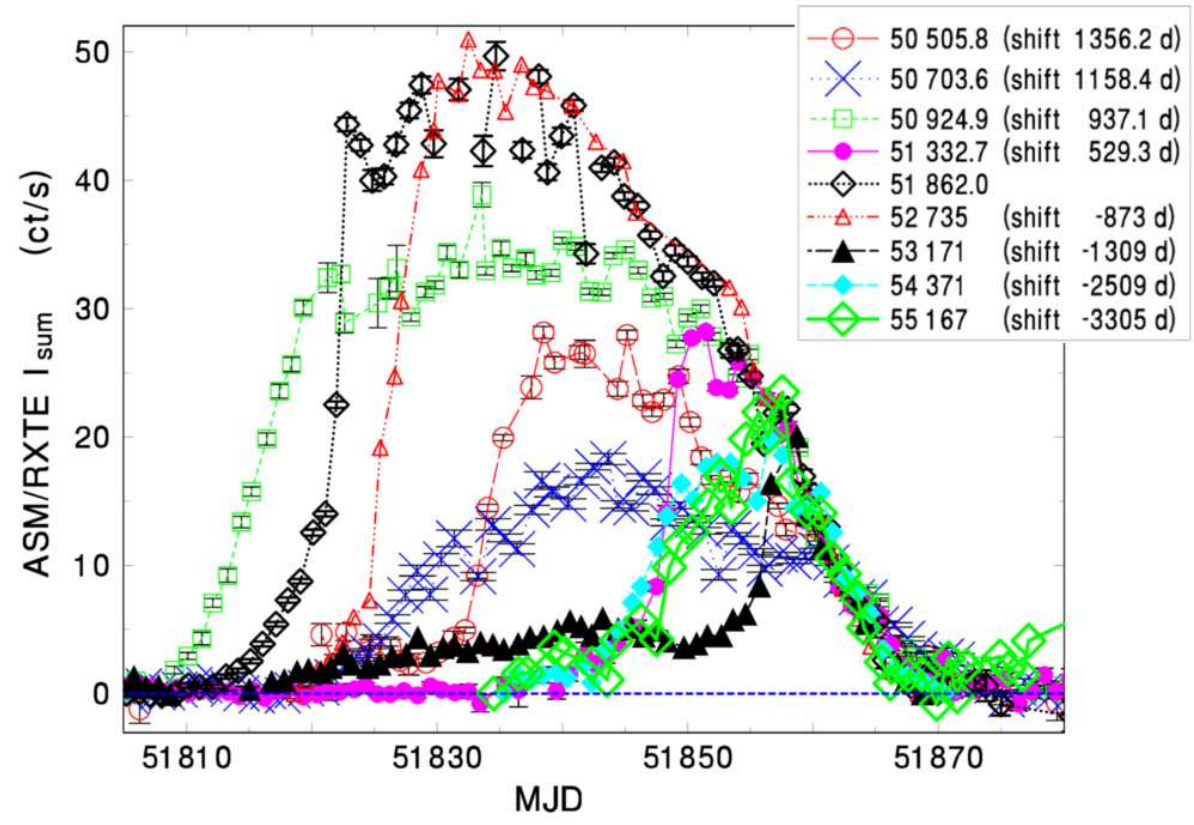

Figure 7: Comparison of the decaying branches of the individual outbursts in Aq1 X-1 (the one-day means of the RXTE/ASM observations). The points are connected by a line to guide the eye. The individual outbursts were shifted along the time axis to match the decaying branch of the template - the time of crossing the flux of $10 \mathrm{ct} \mathrm{s}^{-1}$ and the shifts with respect to the template are listed.

\section{Conclusions}

The observed properties of the light curves suggest the large structural changes of the emitting region(s) during the outbursts both of DNe and SXTs. The disk zone where heating front starts changes for the individual outbursts even in a given binary. The observations show that the decaying branch (governed by propagation of cooling front) reproduces well for the individual outbursts both in DNe and SXTs.

The energy output (e.g. fluence) sometimes largely differs even for the neighboring outbursts. Just a small fraction of the disk mass is accreted during outburst but this difference of the energy output does not influence the length of $T_{\mathrm{C}}$. The properties of the disk in the cool state thus play a big role.

The length of $T_{\mathrm{C}}$ in a given DN or SXT is largely variable, but the occurrence of these outbursts is not dramatically irregular. The profile of the $\mathrm{O}-\mathrm{C}$ diagram of $T_{\mathrm{C}}$ shows only small epoch-toepoch fluctuations. The long-term trends can be clearly resolved in the $\mathrm{O}-\mathrm{C}$ diagrams of $T_{\mathrm{C}}$.

The observations speak in favor of an analogy of an SXT with the ZCam-type CVs. This suggests that the accretion disk of some SXTs can reside in the state which is only intermittently unstable (transitions between the segments of a hot thermally stable (in standstills) and unstable disk (outbursts)).

Acknowledgments This study was supported by grant 3-33324S provided by the Grant Agency of the Czech Republic. This work was also supported by the project RVO:67985815. This research 

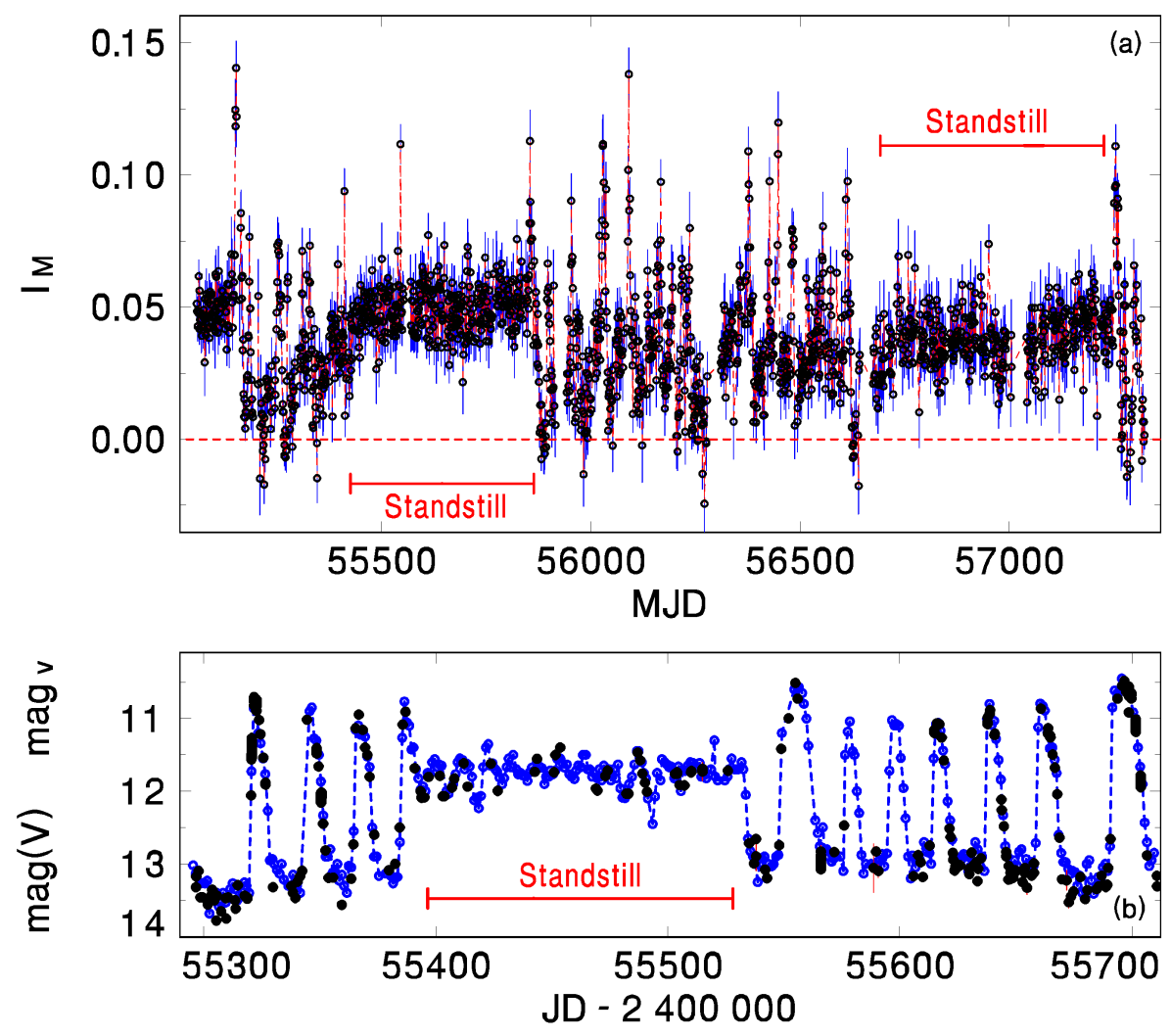

Figure 8: (a) Segment of the light curve of HETE J1900.1-2455 in the 2-4 keV band (ISS/MAXI data). (b) Segment of the optical light curve of Z Cam (the AAVSO data [14]). The closed circles represent CCD measurements $(\operatorname{mag}(V))$ while the open circles mark the one-day means of the visual observations $\left(\operatorname{mag}_{v}\right)$. Standstills are marked for both systems. The points are connected by a line to guide the eye.

has made use of the observations provided by the ASM/RXTE team and the MAXI team. This research has also made use of the observations from the Catalina Transient Survey, AAVSO International database (USA) and the AFOEV database (France). I thank the variable star observers worldwide. I also thank Prof. Petr Harmanec for providing me with the code HEC13. The Fortran source version, compiled version and brief instructions on how to use the program can be obtained at http://astro.troja.mff.cuni.cz/ftp/hec/HEC13/

\section{References}

[1] J. Bailey, Journ. Brit. Astr. Ass., 86, 30 (1975).

[2] C. Chevalier, \& S.A. Ilovaisky, A\&A, 251, L11 (1991).

[3] C. Chevalier, et al., A\&A, 347, L51 (1999).

[4] D. Crampton, et al., ApJ, 300, 788 (1986).

[5] A. J. Drake, et al., ApJ, 696, 870 (2009).

[6] G. Dubus, et al., A\&A, 373, 251 (2001).

[7] H. W. Duerbeck, SSRv, 45, 1 (1987). 
[8] H. W. Duerbeck \& W. C. Seitter, Physics of Classical Novae. Proceedings of Colloquium No.122 of the International Astronomical Union, held in Madrid, Spain, on June 27-30, 1989. Editors, A. Cassatella, R. Viotti; Publisher, Springer-Verlag, Berlin, Germany; New York, NY. Part of the Lecture Notes in Physics book series (1990) (LNP, Vol. 369, p. 165)

[9] J.-M. Hameury, et al.,MNRAS, 298, 1048 (1998).

[10] P. Harmanec, 1992, http://astro.troja.mff.cuni.cz/ftp/hec/HEC13/

[11] A. Henden, Obs. from the AAVSO Int. Database, www.aavso.org (2015).

[12] R. Hudec, Bull. Astron. Inst. Czechosl., 32, 93 (1981).

[13] P. Kaaret, et al., ApJ, 638, 963 (2006).

[14] S. Kafka, Obs. from the AAVSO Int. Database, www.aavso.org (2017).

[15] S. Kitamoto, et al., ApJ, 403, 315 (1993).

[16] K. Koyama, et al., ApJ, 247, L27 (1981).

[17] B. V. Kukarkin, et al., Second Suppl. to the Third Edition of GCVS, Nauka Publishing House, Moscow (1974).

[18] H. Leavitt, Harv. Circ. 142 (1908).

[19] A. M. Levine, et al., ApJ, 469, L33 (1996).

[20] W. H. G.Lewin, et al., X-Ray Binaries, Camb. Astr. S., Vol.26 (1995).

[21] J. Patterson, et al., ApJ, 392, 233 (1992).

[22] W. C. Priedhorsky, \& J. Terrell, ApJ, 280, 661 (1984).

[23] F. Sabbadin, A. Bianchini, A\&AS, 54, 393 (1983).

[24] J. Shears, G. Poyner, JBAA, 120, 169 (2010).

[25] J. Smak, AcA, 34, 161 (1984).

[26] V. Šimon, $A \& A$, 354, 103 (2000a).

[27] V. Šimon, $A \& A, \mathbf{3 6 0}, 627$ (2000b).

[28] V. Šimon, $A \& A, \mathbf{3 8 2}, 910$ (2002).

[29] V. Šimon, $A \& A$, submitted (2017).

[30] P. Szkody, J.A. Mattei, PASP, 96, 988 (1984).

[31] P. Szkody, et al., PASP, 97, 264 (1985).

[32] J.R. Thorstensen, AJ, 91, 940 (1986).

[33] J. R. Thorstensen, C. J. Taylor, MNRAS, 312, 629 (2000).

[34] R. Vanderspek, et al., ATel, 516, 1 (2005).

[35] N. Vogt, $A \& A, \mathbf{8 8}, 66$ (1980).

[36] J. Vondrák, Bull. Astron. Inst. Czechosl., 20, 349 (1969).

[37] B. Warner, Cataclysmic Variable Stars, Cambridge Univ. Press, Cambridge (1995).

[38] M. G. Watson, et al., MNRAS, 212, 917 (1985).

[39] E. Whittaker, \& G. Robinson, The Calculus of Observations, Blackie \& Son Ltd, London, pp. 303-316 (1946). 